\title{
Quality of life and factors affecting it in patients with Alzheimer's disease: a cross- sectional study
}

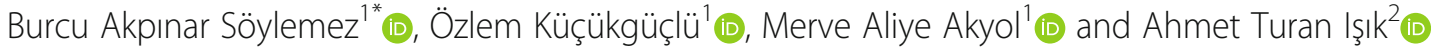

\begin{abstract}
Background: Quality of life $(\mathrm{Q} O \mathrm{~L})$ is a growing area of interest in dementia research. However, it remains a controversial topic. This study aimed to determine the QoL of people with Alzheimer's disease (PWAD) and investigate the factors affecting patients' and caregivers' QoL scores.

Methods: A cross-sectional study design was used. A total of 98 home-dwelling PwADs and their primary caregivers were recruited in the study. Sociodemographic characteristics and QoL scores, activities of daily living $(A D L)$ and instrumental $A D L(I A D L)$, Mini-mental State Examination (MMSE) scores, neuropsychiatric inventory (NPI), and NPI-distress were determined to assess the relevant outcomes. All statistical analyses were performed using SPSS version 22.0. Descriptive statistics, t-test, Pearson correlation, and multinomial regression were used for analysis.

Results: The patients' ratings of their QoL were higher than those of the caregivers. Caregiver education, patients' $A D L$, and IADL were associated with the patients' score on the Quality of Life in Alzheimer's Disease (QoL-AD) scale. In addition to these variables, MMSE, NPI, and NPI-distress were associated with the caregiver scores on QoL-AD.

Conclusion: From a clinical point of view, the proxy-rated scores of QoL cannot replace the self-ratings of the patients. This study suggests that both self- and proxy-rated QoL scores should be applied whenever possible. Focusing on the management of behavioral problems and supporting functionality and cognitive functions may be modifiable factors that may represent targets for intervention to improve the QoL. The findings of this study should also be used to design caregiver educational programs about the determinants of QoL.
\end{abstract}

Keywords: Alzheimer's disease, Quality of life, Caregivers, Proxy

\section{Background}

Alzheimer's disease (AD), the most common type of dementia, is an important health problem in aging populations $[1,2]$. Although the quality of life $(\mathrm{QoL})$ is a growing area of interest in dementia research, it remains a controversial topic. The QoL of people with AD (PwAD) is considered an individual, subjective, dynamic, multidimensional,

\footnotetext{
* Correspondence: burcu.akpinar@deu.edu.tr

1 Department of Internal Medicine Nursing, Dokuz Eylul University Faculty of Nursing, Inciralti, Izmir, Turkey

Full list of author information is available at the end of the article
}

and complex construct and includes the assessment of and adaptation to the consequences of $\mathrm{AD}$ [3].

Measuring the QoL of PwAD is a controversial issue given that the disease progresses with cognitive impairment. Several researchers have reported that PwAD have limited quantification of QoL due to their cognitive impairment and noncognitive symptoms, such as depression and psychosis $[4,5]$. On the other hand, patients should be the main source of information about their own lives, and evidence indicates that PwAD can also comment on their own QoL [6-9]. For this reason, researchers are

(c) The Author(s). 2020 Open Access This article is licensed under a Creative Commons Attribution 4.0 International License, which permits use, sharing, adaptation, distribution and reproduction in any medium or format, as long as you give appropriate credit to the original author(s) and the source, provide a link to the Creative Commons licence, and indicate if changes were made. The images or other third party material in this article are included in the article's Creative Commons licence, unless indicated otherwise in a credit line to the material. If material is not included in the article's Creative Commons licence and your intended use is not permitted by statutory regulation or exceeds the permitted use, you will need to obtain permission directly from the copyright holder. To view a copy of this licence, visit http://creativecommons.org/licenses/by/4.0/. The Creative Commons Public Domain Dedication waiver (http://creativecommons.org/publicdomain/zero/1.0/) applies to the data made available in this article, unless otherwise stated in a credit line to the data. 
searching for ways to measure the QoL meaningfully and accurately in PwAD. A recent review has identified nine QoL measures for people with dementia and has assessed their psychometric properties [10]. Most measures were based on proxy assessment, with questionable validity for people with mild to moderate dementia. The best researched measure was the Quality of Life in Alzheimer's Disease (QoL-AD) scale [9].

This scale is available as a self-rating version [QoL-AD Self-Rating (QoL-AD-SR) scale] and as a proxy rating version (QoL-AD Proxy Rating (QoL-AD-PR) scale). The instrument has been translated in various languages, and the Turkish version has good psychometric properties overall [11].

Discrepancies exist between patients and caregivers regarding the QoL of PwAD, and factors have been associated with divergent ratings [12, 13]. Not only the patients' but also the relatives' and professionals' perceptions of the patients' QoL should be considered [14]. The factors associated with the QoL-AD by selfand proxy ratings have been reported in many recent studies [15-20]; however, none of them have used a Turkish sample. One meta-analysis pointed out that future work should explore whether PwAD in lowerincome countries or other cultures can identify different factors [21]. Testing a subjective concept, such as QoL-AD, as well as the factors associated with QoL$\mathrm{AD}$ in different cultures and in different sociodemographic characteristics will contribute toward shaping an appropriate care based on culture. Researchers should consider that QoL-AD can have cultural differences. In addition to this, improving the QoL is an important focus of various therapeutic interventions as an outcome measure to evaluate interventions for Alzheimer's care [22].

Therefore, this study aimed to investigate the mutual relationships among cognitive impairment, behavioral symptoms, functional abilities, and QoL as evaluated in PwAD and their proxies as well as to identify the factors affecting self' and proxies' QoL ratings.

\section{Methods}

\section{Participants}

A cross-sectional and correlational design was used. Sample selection was conducted using nonprobability convenience sampling. A total of 98 dyads of persons with mild to moderate $\mathrm{AD}$ who were all homedwelling as well as their primary caregivers were recruited in this study. The National Institute of Neurological and Communicative Disorders and Stroke and the Alzheimer's Disease and Related Disorders' Association criteria were used for the diagnosis of probable $\mathrm{AD}$ [23]. The inclusion criteria were as follows: a family member of the patient and the primary person responsible for caregiving and providing care for at least 6 months. The exclusion criteria for the caregivers were those with visual, hearing, or speech impairments. PwAD could be taking cholinesterase inhibitors, memantine and cholinesterase inhibitors, or memantine alone and have a Mini-mental State Examination (MMSE) score of 10 and higher. Patients with severe dementia; those with psychiatric disorders, such as severe depression, schizophrenia, and bipolar disorder; those with other neurological diseases and other types of dementia (such as vascular dementia, multi-infarct dementia, or Lewy body dementia); and uncontrolled clinical problems, such as hypertension, diabetes, were excluded. In the current study, because the PwADs with MMSE score of 10 and higher could usually complete the scale without problems, patients with severe dementia were excluded. The power of the study was evaluated using G. Power 3.1. For QoL-AD-PR, a sample size of 98, effect size of 0.44 , and alpha value of 0.05 were considered, and the power of study was found to be 0.99 . For QoL-AD-SR, a sample size of 98, effect size of 0.18 , alpha value of 0.05 were considered, and the power of study was found to be 0.82 . Generally, a power of 0.80 is acceptable for such studies.

\section{Questionnaires \\ Participant information form}

The form was prepared to obtain the sociodemographic information (such as gender, age, years of education, caregiving period, and relationship) of PwAD and their caregivers. It was filled by caregivers.

\section{QoL-AD}

This scale is available in two parts: QoL-AD-SR and QoL-AD-PR. QoL-AD-SR was completed by patients and QoL-AD-PR was completed by caregivers. The scale contains 13 items concerning physical health, energy, mood, living situation, memory, family, marriage, friends, the individual as a whole, ability to perform chores, ability to conduct recreation activities for fun, money, and life as a whole. The 13 domains are rated as poor (1), fair (2), good (3), or excellent (4), and the total score ranges from 13 to 52 [9]. A high score indicates good QoL. The questions of the scale are simple, clear, and understandable for easy understanding of PwAD who have cognitive failure [9]. The scores are calculated separately for self-reported and proxy ratings. The total score is calculated by multiplying the score obtained through the answers of patients by two, adding the score of caregivers, and then dividing the answer by three [9]. Cronbach's alpha values for the self-reported and proxy ratings for the Turkish population were in the high range (0.84 and 0.77 , respectively) [11]. 


\section{CDR scale}

This scale was used to determine the possible stages of cognition and function: 0 (no dementia), 0.5 (questionable dementia), 1 (mild dementia), 2 (moderate dementia), and 3 (severe dementia) [24]. It was determined by the physician (fourth researcher) in the research team.

\section{MMSE}

MMSE comprises items regarding orientation, learning, short-term memory, language use, comprehension, and basic motor skills. It is used to assess the cognitive function. The total score ranges from 0 to 30 . A low score indicates high cognitive impairment [25].

\section{$A D L$}

The ability of PwAD to perform functional activities was assessed with Barthel ADL and Lawton's IADL [26, 27].

\section{NPI}

NPI was used to investigate the presence and severity of neuropsychiatric symptoms; the scores range from 0 to 144 , with high scores corresponding to severe behavioral disorders [28].

\section{NPI-distress}

The NPI has a distress section to evaluate caregiver distress toward behavioral symptoms. The distress of care was assessed as perceived by the caregivers of PwAD. The total score ranges from 0 to 60 . A high score indicates a high level of perceived distress.

\section{Procedures}

The study was conducted between June 2017 and July 2018 at a dementia outpatient clinic in the west part of Turkey. The data were collected during follow up of the patients at the outpatient clinic. The administration of the questionnaire lasted for $25 \mathrm{~min}$. Caregivers filled the scale by themselves considering the QoL-AD they care for. PwAD responded to questions about their QoL by verbally.

Approval to conduct the study was obtained from the ethical committee of the Dokuz Eylul University, Noninvasive Research Ethics Board (2017/21-45). The participants who agreed to participate in the study (after written permission had been obtained) were asked to complete the questionnaires. PwAD and their caregivers had a face-to-face contact with the researcher.

\section{Statistical analyses}

All statistical analyses were performed using SPSS version 22.0. The Shapiro-Wilk test was used to verify the normal distribution of variables. Parametric variables were described by their mean and standard deviation
(SD) and nonparametric variables were described by their median and interquartile ranges.

For the QoL-AD scores, the average of the selfreported and proxy scores were compared using the student's t-test. Correlational analyses (Pearson's r) were performed to clarify the relationships among cognitive impairment, behavioral symptoms, functional abilities, caregiver distress, and QoL. The stepwise forward multiple linear regression analysis was performed to identify the optimal model of predictors that explain the outcome variable of interest. The "enter" method, in which all the variables are included in the model, was used. The variance inflation factor (VIF) and tolerance were used to detect multicollinearity between the independent variables in the regression model. The independent variables with VIF $>10$ were removed from the model, and tolerance was less than 0.20 . For all analyses, the level of statistical significance was set at $p \leq 0.05$.

\section{Results}

A total of 98 home-dwelling patients were included. The mean ages of patients and caregivers were 73.58 $(\mathrm{SD}=9.37)$ and 56.85 years $(\mathrm{SD}=13.64)$, respectively. A total of 65 patients $(66.3 \%)$ and 58 caregivers $(59.2 \%)$ were women. Among the caregivers, 52 (53.1\%) were the children of the patients and 46 (46.9\%) were spouses.

The mean MMSE score was $17.38 \pm 4.31$, and $68.4 \%$ of patients had mild AD. The mean NPI and NPI-distress scores were $22.86 \pm 18.82$ and $11.85 \pm 9.52$, respectively. Table 1 presents the sociodemographic and clinical characteristics of the study population.

Comparisons of the total QoL-AD scores between patients $(38.30 \pm 4.26)$ and caregivers $(31.58 \pm 4.85)$ revealed a statistically significant difference $(t=64.40$, $p<0.001)$. The patients rated a higher QoL than the caregiver group.

No statistically significant difference was observed between the QoL assessments of patients who are cared for by spouses and children ( $t=0.801, p=0.090)$.

Self-reported QoL score was the only sociodemographic variable that showed correlation with caregiver education $(p=0.001)$. No significant differences were observed in age, gender, and care duration. No significant differences were noted the in self-reported QoL scores across different CDR stages, whereas caregivers reported poor QoL in the moderate stage (Table 2).

Pearson correlation coefficient revealed that PwAD self-rated QoL showed a positive relationship with IADL $(p=0.020)$ and ADL $(p=0.012)$. Pearson correlation coefficient also revealed that the proxy-rated QoL of the patients showed a positive relationship with IADL $(p<$ $0.001)$, ADL $(p=0.023)$, and MMSE $(p<0.001)$. A negative relationship was observed between NPI total score 
Table 1 Sociodemographic and clinical characteristics of participants

\begin{tabular}{|c|c|c|}
\hline & Mean & SD \\
\hline \multicolumn{3}{|l|}{ Patient characteristics } \\
\hline Gender Female (\%) & 66.3 & \\
\hline CDR (\%): $1 / 2$ & $68.4 / 31.6$ & \\
\hline Age & 73.58 & 9.37 \\
\hline Years of education & 8.03 & 4.54 \\
\hline MMSE & 17.38 & 4.31 \\
\hline NPI & 22.86 & 18.82 \\
\hline ADL & 91.02 & 15.43 \\
\hline IADL & 4.59 & 2.69 \\
\hline NPI-Distress & 11.85 & 9.52 \\
\hline QoL-AD-SR & 38.30 & 4.26 \\
\hline \multicolumn{3}{|c|}{ Caregiver characteristics } \\
\hline Gender Female (\%) & 59.2 & \\
\hline Age & 56.85 & 13.64 \\
\hline Years of education & 11.75 & 4.91 \\
\hline Caregiving period & 3.03 & 2.41 \\
\hline \multicolumn{3}{|c|}{ Relationship with the patient } \\
\hline Child (\%) & 53.1 & \\
\hline Spouse (\%) & 46.9 & \\
\hline QoL-AD-PR & 31.58 & 4.85 \\
\hline
\end{tabular}

Abbreviations: $A D L$ Activities of Daily Living, IADL Instrumental ADL, MMSE Mini-mental State Examination, NPI Neuropsychiatric Inventory, QoL-AD Quality of Life in Alzheimer's Disease, QoL-AD-SR QoL-AD Self-Rating, QoL-AD-PR QoL-AD Proxy Rating, CDR Clinical Dementia Rating

$(p<0.001)$ and NPI-distress $(p<0.001)$ and the proxyrated QoL (Table 2).

This study also aimed to determine the most important predicting variables that explain the largest proportion of variance of self- and proxy-rated QoL. The detailed results

Table 2 Correlation of the QoL-AD-SR and QoL-AD-PR with study variables

\begin{tabular}{|c|c|c|c|c|}
\hline \multirow[t]{3}{*}{ Variable } & \multirow{2}{*}{\multicolumn{2}{|c|}{$\begin{array}{l}\text { Self-score } \\
\text { QOL-AD }\end{array}$}} & \multirow{2}{*}{\multicolumn{2}{|c|}{$\frac{\text { Proxy-score }}{\text { QOL-AD }}$}} \\
\hline & & & & \\
\hline & $r$ & $p$ & $\mathbf{r}$ & $p$ \\
\hline QOL-AD-SR & 1.00 & .001 & .377 & .001 \\
\hline QOL-AD-PR & .377 & .001 & 1.00 & .001 \\
\hline Caregiver education & .33 & .001 & .237 & .019 \\
\hline $\mathrm{ADL}$ & .25 & .012 & .238 & .018 \\
\hline IADL & .235 & .020 & .443 & .001 \\
\hline MMSE & .165 & .104 & .400 & .001 \\
\hline NPI & -.007 & .949 & -.532 & .001 \\
\hline NPI-Distress & -.057 & .576 & -.571 & .001 \\
\hline
\end{tabular}

are shown in Table 3. Analysis revealed that caregivers' education years, ADL, and IADL explained $12 \%$ of the variance in self-rated QoL, whereas caregivers' education years, ADL, IADL, MMSE, NPI, and NPI-distress explained 26\% of the variance in proxy-rated QoL (Table 3).

\section{Discussion}

In this cross-sectional and prospective study, the caregivers' education, ADL, and IADL of the PwAD were associated with the QoL-AD-SR. Additionally, MMSE, NPI, and NPI-distress were associated with the QoLAD-PR scores.

Our analysis showed no differences in terms of gender of the informal caregiver, as stated by Römhild et al. [17], Schumann et al. [15] and Martyr [18]. However, this result was different from the findings of Conde-Sala et al. [13]. The objective of the study by Conde-Sala et al. (2014) was to analyze burden and mental health in the caregiver subgroups according to gender and relationship to the patient (husbands, wives, sons, and daughters). It could be possible that previous relationships with the patient can affect this result.

In the current study, the relationship with informal caregiver showed no influence on PwAD, similar to the findings of Huang et al. [29] and Römhild et al. [17]. Unlike the findings of Robertson et al. [16], ve Schumann et al. [15] concluded that the average QoL-AD score of the caregivers as the spouse of patients were higher than that of the children [30,31]. The lack of difference in our study was attributed to culture and the commitment of the children toward their parents. This result might be due to strong family bonds and the sensibility of caregiving for the PwAD at home in the Turkish society.

Table 3 Regression models of factors predicting self-rated Qol, proxy-rated QoL

\begin{tabular}{lllll}
\hline & Beta coefficient & $\mathbf{R}^{\mathbf{2}}$ & Adjusted $\mathbf{R}^{\mathbf{2}}$ & $\boldsymbol{P}$ value \\
\hline Self-rated QoL & & & & \\
$\quad$ Caregiver education & $.333(p=.001)$ & & & \\
ADL & $.252(p=.006)$ & .149 & .122 & $<.010$ \\
IADL & $.235(p=.001)$ & & & \\
Proxy-rated QoL & & & & \\
Caregiver education & $.237(p=.009)$ & & \\
MMSE & $.400(p=.001)$ & .306 & .260 & $<.010$ \\
ADL & $.238(p=.009)$ & & & \\
IADL & $.443(p=.001)$ & & & \\
NPI & $-.402(p=.001)$ & & \\
NPI-Distress & $-.404(p=.001)$ & & \\
\hline
\end{tabular}

Note: Non-significant variables for self-rated QoL: MMSE, NPI, NPI-Distress Abbreviations: ADL Activities of Daily Living, IADL Instrumental ADL, MMSE Mini Mental State Examination, NPI Neuropsychiatric Inventory, QoL-AD Quality of Life in Alzheimer's Disease, QoL-AD-SR QoL-AD Self-Rating, QoL-AD-PR QoL-AD Proxy Rating 
According to the results of this study, self- and proxyrated QoL are not related to age. This result is similar to that of Römhild et al. [17]. In a recent systematic review and correlational meta-analysis by Martyr et al. [18], age was not associated with the QoL. However, Barbe et al. [20] and Schumann et al. [15] have stated that age has an inverse relationship with the QoL. In a study conducted by Barbe et al. [20], the mean age of patient was 82 years (it was 73 years in the current study). This could have had an effect on the lower QoL. In consequence of using the cohort method in Schumann et al. [15], the results can be different with the current study.

\section{Differences between self and proxy QoL of AD}

Patients rated a significantly higher QoL than the caregivers, which is in accordance with the results of previous studies [12, 13, 15-20]. As stated by other researchers, this situation is thought to be due to patients' insufficient insight, awareness of caregivers about their patients' condition, and negative outcomes such as burden of care. Disease severity affects caregivers more than the patients themselves [32]. In addition, patients having reduced abilities to judge their own difficulties due to increased cognitive impairment may affect this situation [12]. In this regard, caregivers may assume that the QoL of the patient with impaired cognition also worsens.

\section{Factors affecting QoL-AD \\ Factors affecting QoL-AD-SR}

According to the results of this research, caregiver education and the functional level of patients affect their self-reported QoL. Unlike this study result, Römhild et al. [17] and Martyr et al. [18] concluded that caregiver education is not related to QoL. Different from the findings in literature, in this study, education in providing care is an important variable in the care of patients and may have affected the QoL positively. Given that educated caregivers are conscious about the approach and care of their patients, this positive approach is thought to enable patients and caregivers to achieve positive QoL.

The ratings of both QoL scores are low if the patient is functionally impaired. Functionality, as stated by Schumann et al. [15], Römhild et al. [17], and Martyr et al. [18], is related to QoL. High self-reported QoL scores indicate an excellent functional status. Functionality is one of the indispensable components of life. The current relation result is logical because the need for functional support generally stems from the presence of multiple functional impairments, and the patient's perception of their own health is heavily influenced by the presence of such functional impairments.

\section{Factors affecting QoL-AD-PR}

Caregiver education years, functionality, cognition, behavioral symptoms, and distress affect proxy-rated QoL. Similar to the finding of this study, Sousa et al. [33] stated that caregiver QoL ratings are influenced by the caregivers' educational level [33]. Educated caregivers who are aware of their patients' condition are thought to have a positive attitude toward the patients' QoL.

High QoL scores are an expected outcome for patients with excellent functional state. It was observed that individuals with high functionality have a high QoL score [18]. A caregiver who sees their loved one suffering from several functional impairments is highly normally expected to rate the patient's QoL more negatively.

In this study, significant correlations were observed between proxy-rated QoL and worsened cognitive impairment. Self-rated patient QoL showed association with the said factor. However, QoL is a controversial topic. The findings of the current study are inconsistent with those of the literature. Conde-Sala et al. [31] stated that no significant correlation exists between patientand caregiver-reported QoL and cognitive function. The results of this study on cognitive function are similar to those of other studies [12, 13, 18].

Caregivers experience difficulty in managing behavioral symptoms. High behavioral symptoms negatively affect proxy-rated QoL. A strong negative relationship exists between behavioral symptoms and proxy-rated QoL [15]. Hongisto et al. [19] examined the effect of NPI on the QoL of PwAD longitudinally over a five-year period. Although the behavioral problems of the patients increased in the five-year period, self-rated QoL showed no change; however, proxy-rated QoL decreased gradually against the increasing behavioral symptoms observed by the caregivers.

In this study, significant correlations were observed between proxy-rated QoL and caregiver distress. The results of this study are similar to those in the literature $[15,17,18,20]$. Using regression analysis, Fuh et al. [34] observed that caregiver distress (NPI-distress) was among the determinants affecting proxyrated QoL.

\section{Regression analysis}

Overall, our fitted regression models accounted for 12 and $26 \%$ of the observed variance of the difference between self-reported and proxy ratings. Therefore, other unknown factors influence the difference between self and proxy ratings, and they were not addressed by the data of our analysis.

\section{Conclusion}

$\mathrm{AD}$ has become a health concern due to its dramatically increasing prevalence. This condition may result in 
serious consequences for patients, their caregivers, and health services. For these reasons, the QoL of PwAD and the factors affecting patients' and caregivers' QoL rating must be determined. Our study highlights the statistically significant different caregiver- and self-rated QoLs. This study showed that the discrepancy between patientand caregiver-reported QoL showed no change based on cultural boundaries. Low self- and proxy-rated QoL ratings were observed with low caregiver education and functionally impaired patients. Proxy-rated QoL was also rated low if the patient had poor cognitive status and behavioral problems as well as if the caregivers had suffered from behavioral problems related to distress.

From a clinical point of view, the proxy-rated QoL rating cannot replace the self-rating of PwAD. This study suggests that both self- and proxy-rated QoL ratings should be applied whenever possible. Focusing on the management of behavioral problems and supporting functionality and cognitive functions may be modifiable factors that may represent targets for intervention to improve the QoL. The findings of this study should be used to design caregiver educational programs about the determinants of QoL.

Several limitations have to be considered in our study. First, the findings are specific to patients with mild to moderate $\mathrm{AD}$ and who live in their homes. Therefore, the results cannot be generalized to people with severe $\mathrm{AD}$ or institutionalized patients. In addition, the emotional aspects of caregivers, such as depression and anxiety, were not evaluated. Future research may focus on QoL changes over time. The QoL of late-stage PwAD and the factors that affect patients' and caregivers' QoL rating must also be determined.

\section{Abbreviations}

AD: Alzheimer's Disease; QoL: Quality of Life; PwAD: People with Alzheimer's Disease; ADL: Activities of Daily Living; IADL: Instrumental ADL; MMSE: Mini Mental State Examination; NPI: Neuropsychiatric Inventory; QoL-AD: Quality of Life in Alzheimer's Disease; QoL-AD-SR: QoL-AD Self-Rating; QoL-ADPR: QoL-AD Proxy Rating; CDR: Clinical Dementia Rating

\section{Acknowledgments}

We would like to acknowledge Professor Doctor Görsev Yener for support during data collection. Again we would like to thank study participants.

\section{Authors' contributions}

BAS wrote the protocol, design study, organized the data collection process, analyzed the data and writing the manuscript. ÖK revise and approve the protocol, reviewing and editing the manuscript. MAA contributed to the data collection, entering of data, writing and drafting the manuscript. ATI revise and approve the protocol, critically reviewed and approved the manuscript for publication. All authors read and approved the final manuscript.

\section{Funding}

This study was financially supported by researchers.

\section{Availability of data and materials}

The datasets used and/or analyzed during the current study are available from the corresponding author on reasonable request by burcu. akpinar@deu.edu.tr

\section{Ethics approval and consent to participate}

The Project was ethically approved by the ethical committee of the Dokuz Eylul University, Noninvasive Research Ethics Board (Turkey) (2017/21-45).

\section{Consent for publication}

Not Applicable.

\section{Competing interests}

The authors have declared that they have no conflicts of interest.

\section{Author details}

'Department of Internal Medicine Nursing, Dokuz Eylul University Faculty of Nursing, Inciralti, Izmir, Turkey. ${ }^{2}$ Department of Geriatrics, Dokuz Eylül University, Faculty of Medicine, İmir, Turkey.

Received: 30 April 2020 Accepted: 1 September 2020 Published online: 10 September 2020

\section{References}

1. Alzheimer's Association. 2020 Alzheimer Facts and Figures: Special report on the front lines: Primary care physicians and Alzheimer's care in America Available from: https://www.alz.org/media/Documents/alzheimers-factsand-figures.pdf. Accessed 30 Apr 2020.

2. Alzheimer's Disease International. The global voice on dementia: World Alzheimer Report 2019: Attitudes to dementia. Available from: https://www. alz.co.uk/research/WorldAlzheimerReport2019.pdf. Accessed 30 Apr 2020.

3. Dichter MN, Schwab CG, Meyer G, Bartholomeyczik S, Halek M. Linguistic validation and reliability properties are weak investigated of most dementia specific quality of life measurements: a systematic review. J Clin Epidemiol. 2016;70:233-45.

4. Bowling A, Rowe G, Adams S, Sands P, Samsi K, Crane M, et al. Quality of life in dementia: a systematically conducted narrative review of dementiaspecific measurement scales. Aging Ment Health. 2015;19:1,13-31.

5. Logsdon RG, Gibbons LE, McCurry SM, Teri L. Assessing quality of life in older adults with cognitive impairment. Psychosom Med. 2002;64:3,510-9.

6. Trigg R, Jones RW, Knapp M, King D, Lacey LA. DADE-2 investigator groups. The relationship between changes in quality of life outcomes and progression of Alzheimer's disease: results from the dependence in $A D$ in England 2 longitudinal study. Int J Geriatr Psychiatry. 2015;30:4,400-8.

7. Akpınar B, Küçükgüçlü Ö. Is quality of life of people with dementia measurable? Deuhyo ED. 2011;4:3,141-3.

8. Smith SC, Murray J, Banerjee S, et al. What constitutes health related quality of life in dementia? Development of a conceptual framework for people with dementia and their carers. Int J Geriatr Psychiatry. 2005;20:889-95.

9. Logsdon RG, Gibbons LE, McCurry SM, Teri L. Quality of life in Alzheimer's disease: patient and caregiver reports. Aging Ment Health. 1999;5:1,21-32.

10. Hughes $L$, Farina N, Page TE, Tabet N, Banerjee S. Psychometric properties and feasibility of use of dementia specific quality of life instruments for use in care settings: a systematic review. Int Psychogeriatr. 2019:1-15. https:// doi.org/10.1017/S1041610218002259.

11. Akpınar B, Küçükgüçlü Ö. The validity and reliability of the Turkish version of the quality of life scale for patients with Alzheimer's disease (QOL-AD). J Neurol Sci (Turkish). 2012;32:554-65.

12. Andrieu S, Coley N, Rolland Y, et al. Assessing Alzheimer's disease patients' quality of life: discrepancies between patient and caregiver perspectives. Alzheimers Dement. 2016;12:4,427-37.

13. Conde-Sala JL, Reñé-Ramírez R, Turró-Garriga O, et al. Severity of dementia, anosognosia, and depression in relation to the quality of life of patients with Alzheimer disease: discrepancies between patients and caregivers. Am J Geriat Psychiat. 2014;22:2,138-47.

14. Mack JL, Whitehouse PJ. Quality of life in dementia: state of the art- report of the international working Group for Harmonization of dementia drug guidelines and the Alzheimer's society satellite meeting. Alzheimer Dis Assoc Disord. 2001;15:2,69-71.

15. Schumann C, Alexopoulos P, Perneczky R. Determinants of self-and carerrated quality of life and caregiver burden in Alzheimer disease. Int I Geriatr Psychiatry. 2019;34:10,1378-85.

16. Robertson S, Cooper C, Hoe J, et al. Comparing proxy rated quality of life of people living with dementia in care homes. Psychol Med. 2020;50:1,86-95.

17. Römhild J, Fleischer S, Meyer G, et al. Inter-rater agreement of the quality of life-Alzheimer's disease (QoL-AD) self-rating and proxy rating scale: 
secondary analysis of right time place care data. Health Qual Life Outcomes. 2018;16:131,1-13.

18. Martyr A, Nelis SM, Quinn C, et al. Living well with dementia: a systematic review and correlational meta-analysis of factors associated with quality of life, well-being and life satisfaction in people with dementia. Psychol Med. 2018:48:13,2130-9.

19. Hongisto K, Hallikainen I, Selander T, et al. Quality of life in relation to neuropsychiatric symptoms in Alzheimer's disease: 5-year prospective ALSOVA cohort study. Int J Geriatr Psychiatry. 2018;33:1,47-57.

20. Barbe C, Jolly D, Morrone I, et al. Factors associated with quality of life in patients with Alzheimer's disease. BMC Geriatr. 2018;18:159,1-9.

21. O'rourke HM, Duggleby W, Fraser KD, Jerke L. Factors that affect quality of life from the perspective of people with dementia: a metasynthesis. J Am Geriatr Soc. 2015;63:1,24-38.

22. National Collaborating Centre for Mental Health. A NICE-SCIE Guideline on supporting people with dementia and their carers in health and social care: National Practive Guideline 2016 Number 42. London: The British Psychological Society \& The Royal College of Psychiatrists; 2016.

23. McKhann G, Drachman D, Folstein M, Katzman R, Price D, Stadlan EM. Clinical diagnosis of Alzheimer's disease: report of the NINCDS-ADRDA work group under the auspices of Department of Health and Human Services Task Force on Alzheimer's disease. Neurology. 1984;34:7,939-44.

24. Hughes CP, Berg L, Danziger WL, Coben L, Martin RL. A new clinical scale for the staging of dementia. Br J Psychiatry. 1982;140:566-72.

25. Folstein MF, Robins LN, Helzer JE. The mini-mental state examination. Arch Gen Psychiatry. 1983:40:7,812

26. Collin C, Wade DT, Davies $S$, Horne V. The Barthel ADL index: a reliability study. Int Disabil Stud. 1988;10:2,61-3.

27. Lawton MP, Brody EM. Assessment of older people: self-maintaining and instrumental activities of daily living. The Gerontologist. 1969;9:3,179-86.

28. Cummings JL, Mega M, Gray K, Rosenberg-Thompson S, Carusi DA, Gornbein J. The neuropsychiatric inventory: comprehensive assessment of psychopathology in dementia. Neurology. 1994;44:12,2308.

29. Huang H-L, Weng $L-C$, Tsai $Y$-H, et al. Predictors of self- and caregiver-rated quality of life for people with dementia living in the community and in nursing homes in northern Taiwan. Int Psychogeriatr. 2015;27:825-36.

30. Conde-Sala JL, Garre-Olmo J, Turro-Garriga O, Vilalta-Franch J, Lopez-Pousa S. Quality of life of patients with Alzheimer's disease: differential perceptions between spouse and adult child caregivers. Dement Geriatr Cogn Disord. 2010;29:2,97-108.

31. Conde-Sala JL, Garre-Olmo J, Turro-Garriga O, Lopez-Pousa S, Vilalta-Franch J. Factors related to perceived quality of life in patients with Alzheimer's disease: the patient's perception compared with that of caregivers. Int J Geriatr Psychiatry. 2009;24:6,585-94.

32. Livingston G, Cooper C, Woods J, Milne A, Katona C. Successful ageing in adversity: the LASER AD longitudinal study. J Neurol Neurosurg Psychiatry. 2008;79:6,641-5.

33. Sousa MFB, Santos RL, Arcoverde C, et al. Quality of life in dementia: the role of non-cognitive factors in the ratings of people with dementia and family caregivers. Int Psychogeriatr. 2013;25:7,1097-105.

34. Fuh J, Wang S. Assessing quality of life in Taiwanese patients with Alzheimer's disease. Int J Geriatr Psychiatry. 2006;21:2,103-7.

\section{Publisher's Note}

Springer Nature remains neutral with regard to jurisdictional claims in published maps and institutional affiliations.

Ready to submit your research? Choose BMC and benefit from:

- fast, convenient online submission

- thorough peer review by experienced researchers in your field

- rapid publication on acceptance

- support for research data, including large and complex data types

- gold Open Access which fosters wider collaboration and increased citations

- maximum visibility for your research: over $100 \mathrm{M}$ website views per year

At BMC, research is always in progress.

Learn more biomedcentral.com/submissions 